\title{
Harmony Search-based Cluster Initialization for Fuzzy C-Means Segmentation of MR Images
}

\author{
Osama Moh'd Alia, Rajeswari Mandava, \\ Dhanesh Ramachandram \\ CVRG - School of Computer Sciences \\ University Sains Malaysia \\ 11800 USM, Penang, Malaysia \\ sm_alia@yahoo.com, \{mandava,dhaneshr\}@cs.usm.my
}

\author{
Mohd Ezane Aziz \\ Department of Radiology - Health Campus \\ Universiti Sains Malaysia \\ 16150 K.K, Kelantan, Malaysia \\ drezane@kb.usm.my
}

\begin{abstract}
We propose a new approach to tackle the well known fuzzy c-means (FCM) initialization problem. Our approach uses a metaheuristic search method called Harmony Search (HS) algorithm to produce near-optimal initial cluster centers for the FCM algorithm. We then demonstrate the effectiveness of our approach in a MRI segmentation problem. In order to dramatically reduce the computation time to find nearoptimal cluster centers, we use an alternate representation of the search space. Our experiments indicate encouraging results in producing stable clustering for the given problem as compared to using an FCM with randomly initialized cluster centers.
\end{abstract}

\section{INTRODUCTION}

Image segmentation is one of the most important and challenging step in digital image analysis. It can be defined as a process of subdividing the digital image into constituent regions; each region has similar features. As image segmentation can be modelled as a clustering problem, several clustering algorithms have been successfully applied in image segmentation [1]. In practice, most data sets exhibit unclear boundaries between clusters. Medical images particularly, often consist of regions with fuzzy and disjointed boundaries. In this application context, fuzzy clustering has shown tremendous potential as it can naturally cope with such data characteristics. It is therefore unsurprising that the fuzzy c-means algorithm (FCM) [2] has found numerous applications in image segmentation problems and produced very good results [3], [4]. However, the basic FCM algorithm has several limitations and has prompted researchers to investigate various improvisations. Specifically, FCM has a tendency to be trapped in local optima and being prone to initialization sensitivity [5], [6]. These issues stem from the fact that FCM exhibits a greedy search behavior [7] that only guarantees to yield local optima. As a consequence, the improper selection of initial cluster centers will generally lead to undesirable clustering results.

One possible approach to obtain a global optimal solution is to combine FCM algorithm with one of the evolutionary (metaheuristic) optimization algorithm. This approach has been extensively proposed in the literature (see Section II). The solutions obtained by evolutionary algorithms are often not necessarily exact solutions to the problems but rather 'good enough' or near-optimal solutions [8].
In this paper, we investigate the effectiveness of the Harmony Search (HS) algorithm to produce near-optimal initial cluster centers for FCM algorithm to guarantee good and stable image segmentation results. HS is a new metaheuristic algorithm, which was developed by Geem [9] and successfully applied to different optimization problems [10]. HS possess several advantages over traditional optimization techniques such as: (1) it is a simple metaheuristic algorithm and does not require initial value settings for decision variables (2) uses stochastic random searches, therefore derivative information is unnecessary (3) has few parameters that require tuning and (4) can be easily adopted for various types of optimization problems [11]. These features prompted us to investigate its effectiveness in optimizing cluster centers for the FCM-based segmentation of MR images.

Our proposed approach consists of two stages. In the first stage, the harmony search algorithm, using a simplified image representation, explores image search space to find the most optimal cluster centers. The cluster centers found by HS are evaluated using a reformulated FCM objective function [12]. In the second stage, those cluster centers are used by FCM as initial cluster centers. The proposed approach is evaluated on two separate medical image datasets. First, a set of simulated MR brain images obtained from McGill University [13] and secondly on a set of pathological osteosarcoma MR images obtained from the Radiology Department at Universiti Sains Malaysia Hospital, Kelantan, Malaysia.

The rest of the paper is organized as follows: Section II discusses the related work. Section III describes fuzzy clustering with FCM. Section IV describes the harmony search algorithm. Section V discusses the proposed algorithm. Section VI shows the experimental results as well the conclusion is presented in the final section.

\section{RELATED WORK}

Within the last decades, several metaheuristic search algorithms have been used to search for optimal cluster centers for FCM algorithm. These algorithms worked in a way that can explore all possible solutions in the problem search space or at least can escape falling in a local optimal solution. These 
algorithms include simulated annealing as in [14], [15], tabu search [16], [17], genetic algorithm [18], bees optimization [19], particle swarm [20], and ant colony algorithm [7].

Recently, Ingram et al. [10] provides a survey on the application of the HS algorithm in various FCM clustering problems. Ayvaz [21] used HS for zone structure and zonal transmissivities for a heterogeneous aquifer, Forsati et al. [22] and Mahdavi et al. [23] for web documents clustering, Wang [24] for fuzzy classifier, and Malaki et al [25] for clustering NASA radiator data. It is evident from literature that HS is efficient in tackling the FCM's initialization problem in different domains. Therefore, we explore HS algorithm to FCM in a medical MR image segmentation domain since this domain is a very complicated domain according to natural difficulties of MR images. Also, according to the authors' knowledge, this is the first effort to apply HS-FCM algorithm to image segmentation.

\section{Clustering with Fuzzy C-Means}

Clustering is a typical unsupervised learning technique for grouping similar data points (patterns) according to some measure of similarity that maximizes the intra-cluster similarity and minimizes the inter-cluster similarity [1]. Clustering algorithm of a fuzzy partitioning type is performed on a set of $n$ objects(pixels) $X=\left\{x_{1}, x_{2}, \ldots, x_{n}\right\}$, each of which, $x_{i} \in \Re^{d}$, is a feature vector consisting of $d$ real-valued measurements describing the features of the object represented by $x_{i}$. Fuzzy clusters $c$ of the objects can be represented by a fuzzy membership matrix called fuzzy partition $U=\left[u_{i j}\right]_{(c \times n)}$ ( $U \in M_{f c n}$ as in Eq. 2). Where $u_{i j}$ represents the fuzzy membership of the $i t h$ object to the $j$ th fuzzy cluster. In this case, every data object belongs to a particular (possibly null) degree of every fuzzy cluster.

FCM is an iterative procedure which is able to locally minimize the following objective function:

$$
J_{m}=\sum_{j=1}^{c} \sum_{i=1}^{n} u_{i j}^{m}\left\|x_{i}-v_{j}\right\|^{2}
$$

where $\left\{v_{j}\right\}_{j=1}^{c}$ are the centroids of the clusters $c .\|$. denotes an inner-product norm (e.g. Euclidean distance) from the data point $x_{i}$ to the $j t h$ cluster center, and the parameter $m \in[1, \infty)$, is a weighting exponent on each fuzzy membership that determines the amount of fuzziness of the resulting classification.

$$
M_{f c n}=\left\{\begin{array}{c}
U \in \Re^{c \times n} \mid \sum_{j=1}^{c} U_{i j}=1,0<\sum_{i=1}^{n} U_{i j}<n \\
\text {, and } U_{i j} \in[0,1] ; 1 \leq j \leq c ; 1 \leq i \leq n
\end{array}\right\}
$$

FCM's steps can be summarized as follows [2]:

1) Select the number of fuzzy clusters, $c$.

2) Select initial cluster centers $v_{1}, v_{2}, \ldots, v_{c}$.

3) Compute the elements of the fuzzy partition matrix:

$$
u_{i j}=\frac{1}{\sum_{k=1}^{c}\left(\frac{\left\|x_{i}-v_{j}\right\|}{\left\|x_{i}-v_{k}\right\|}\right)^{\frac{2}{m-1}}}
$$

4) Compute the cluster centers:

$$
v_{j}=\frac{\sum_{i=1}^{n} u_{i j}^{m} \cdot x_{i}}{\sum_{i=1}^{n} u_{i j}^{m}}
$$

5) Repeat steps 3 and 4 until the number of iterations $t$ exceeds a given limit or a termination criterion is satisfied:

$$
\left\|v_{\text {new }}-v_{\text {old }}\right\|<\varepsilon
$$

where $\varepsilon<0.001$

\section{HARMONy SEARCH Algorithm}

It is a relatively new metaheuristic algorithm developed by Geem et al. in 2001 [9] for optimization problems. It is a very successful metaheuristic algorithm through its ability to exploit the new suggested solution (harmony) synchronizing with exploring the search space in both intensification and diversification parallel optimization environment [26]. This algorithm imitates the natural phenomenon of musicians' behavior when they cooperate the pitches of their instruments together to achieve a fantastic harmony as measured by aesthetic standards. The following description of HS as in [26]:

HS idealizes the improvisation process by a skilled musician. HS imitates the three possible choices for musician when they improvise: (1) playing any famous tune exactly from his or her memory; (2) playing something similar to the aforementioned tune (thus adjusting the pitch slightly); or (3) composing new or random notes. The three corresponding components in HS become: usage of harmony memory (HM), pitch adjusting, and randomization respectively.

$\mathrm{HM}$ is used as a correspondent step when the musician uses his or her memory to generate an excellent tune. This important step ensures that good harmonies are considered as elements of new solution vectors. It is a cumulative process. Furthermore, pitch adjusting and randomization are used efficiently In order to improvise new solution vectors.

After harmony memory size (HMS) is set by user, an initialization step for HM is considered with randomly generated feasible solutions. Each row of HM consists of one candidate solution.

The new harmony (solution vector) will be generated depending on HS's improvising rules, this new harmony will inherit the values of its components from HM solution rows stored in HM with probability of Harmony Memory Consideration Rate $(\mathrm{HMCR}) \in[0,1]$. This adopted parameter by $\mathrm{HS}$ is to provide an efficient mechanize of using stored solutions in harmony memory. Otherwise, the value of the components of the new solution is selected randomly from the possible range with probability (1-HMCR). This step is called a randomization, which increase the diversity of the solutions and drive the system further to explore various diverse solutions to attain the global optimality. Furthermore, the new solution components that selected out of a memory consideration operator are examined to be pitch adjusted with probability of Pitch Adjustment Rate (PAR) $\in[0,1]$. This parameter simulates the music means "changing of the 
frequency', and it means generating a slightly different value for the new harmony vector component as in Eq. (6). This parameter explores some more extra solutions in search space.

$$
\left(a_{i}^{N E W}\right)=\left(a_{i}^{N E W}\right) \pm \operatorname{rand}() * b w
$$

Here, $b w$ is an arbitrary distance bandwidth used to improve the performance of HS and in this paper it is set to $b w=$ $0.0001 * \max \operatorname{Value}(n)$; $\operatorname{rand}()$ is a generated random number $\in[0,1]$.

After the new solution vector is generated, a comparison step is taken with the worst HM solution in terms of fitness function. If it is better, the new solution is included into the $\mathrm{HM}$ and the worst harmony is excluded.

\section{A Harmony Search for Clustering Problem}

In this section, we describe how the harmony search tackles the problem of clustering, and also shows how image representation reduces the time complexity of our proposed algorithm.

\section{A. Image Representation}

The clustering methods are time consuming when it is performed on a set with $n$ large number of objects [27]. For that, a simplification step is necessary to improve the performance of HS through reducing number of objects to be clustered and therefore reduce the time required to reach the near optimal solution. The simplification process is based on finding the frequency of occurrence of each pixel in the tested image (like a histogram). Therefore, the image is represented in a model such as $X=\left(\left(x_{1}, h_{1}\right), \ldots,\left(x_{i}, h_{i}\right), \ldots,\left(x_{q}, h_{q}\right)\right)$ where $h_{i}$ is the frequency of occurrence $x_{i}$ in the image, and $q$ is the total number of distinct $x$ value in the image with $\left(\sum_{i=1}^{q} h_{i}=n\right)$. As a consequence, the dimensions of partition matrix will be reduced. To illustrate this idea, assume a gray image with 8 -bit resolution and size of $512 \times 512$. Typically there are only 256 possible values for each pixel. Therefore, the value of $n$ becomes 256 instead of 262144 (i.e. $512 \times 512$ ) and the partitioning matrix becomes $U=c \times 256$ instead of $U=c \times 262144$.

\section{B. Harmony Search for Clustering}

In order to choose a suitable cluster centers representation for HS, each harmony memory vector (HMV) encodes candidate cluster centers. Each vector has a physical length of $(c \times d)$. The solution vector will be as in Eq. (7):

$$
H M V=(\overbrace{a_{1} a_{2} \cdots a_{d}}^{v_{1}} \overbrace{a_{1} a_{2} \cdots a_{d}}^{v_{2}} \cdots \overbrace{a_{1} a_{2} \cdots a_{d}}^{v_{c}})
$$

where $a_{k}$ is a decision variable and $a_{k} \in A$, and $A$ is the set of possible range of decision variables which is in image case is image depth. For example, if a gray image has 3 different regions (e.g. white matter, gray matter, and CSF in brain MR image) with 8 bit depth and a 3 features that describe each pixel, then the possible range of decision variables is 0 to 255 and the harmony vector could be like
$(10,30,180,30,45,201,96,140,75)$, where $(10,30,180)$ represent the cluster center values for the first image region, and $(30,45,201)$ represent the cluster center values for the second image region, and so on.

In the initialization step of HM, each decision variable in each solution vector in HM will be initialized randomly from its data range. This step take turn after HS's parameters (HMS, HMCR, PAR, NI) is set. After the HM is filled with initialized solution vectors as in Eq. (8), the fitness value will be calculated for each solution vector in HM by HS objective function (as explained in Section V-C) and then HM vectors will be rearranged in decreasing manner.

$$
H M=\left(\begin{array}{cccc|c}
a_{1}^{1} & a_{2}^{1} & \ldots & a_{N}^{1} & f\left(a^{1}\right) \\
a_{1}^{2} & a_{2}^{2} & \ldots & a_{N}^{2} & f\left(a^{2}\right) \\
\vdots & \vdots & \ldots & \vdots & \vdots \\
a_{1}^{H M S} & a_{2}^{H M S} & \ldots & a_{N}^{H M S} & f\left(a^{H M S}\right)
\end{array}\right)
$$

Now after HM is initialized, HS algorithm start working and for each iteration, a new solution vector is generated according to the improvisation strategy in HS until the stopping criterion is reached. Through this process, the fitness value of each new vector will be calculated and compared with the worst fitness value in harmony memory. Hence, if the new vector's fitness value is better or equal than that worst value in HM, replacement will take place and this new vector will be as a new vector in the HM, otherwise it will be ignored.

Once the HS has met the stopping criterion, a selection of a solution vector from HM with minimum objective function value will take a place and considered as initial centroids for FCM.

Now, after HS algorithm finds near optimal cluster centers that guarantee FCM will not be trapped in a local optima and reaches near global optima, we perform an enhancement to FCM algorithm and that would render the traditional method of multiple random initialization selection unnecessary. We explain our enhancement to FCM in the following sections.

\section{Objective Function}

Since only cluster centers will be used within the HS, a reformulated version of standard FCM's objective function Eq. (9) is considered in this study. Hathaway and Bezdek [12] proposed this reformulated version of the objective function without using the membership matrix $U$ in its calculations but with necessary conditions satisfied by $U$. Although both objective functions (conventional and reformulated) are equivalent but the reformulated version is less complex [12]. Therefore, the computational time for calculating the objective function for each solution vector in HS is reduced.

$$
R_{m}=\sum_{l=1}^{q}\left(\sum_{j=1}^{c} D_{j l}^{\frac{1}{1-m}}\right)^{1-m}
$$

where $D_{i}$ is $\left\|x_{l}-v_{i}\right\|$ the distance from data point $x_{l}$ to the $j$ th cluster center. HS will minimize this value of $R_{m}$ to reach the near optimal solution or meet stopping criterion. 


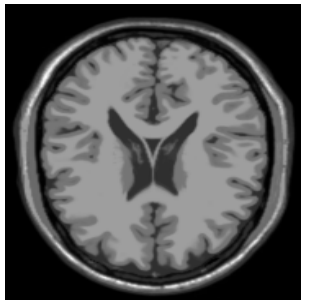

(a)

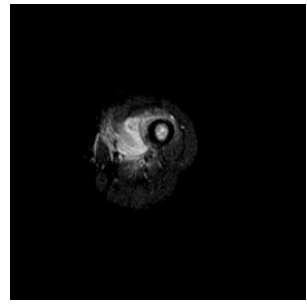

(b)
Fig. 1. Example of MR images. (a) Simulated T1W1 brain MR image with $0 \%$ noise and $0 \%$ intensity inhomogeneity, (b) STIR osteosarcoma MRI.

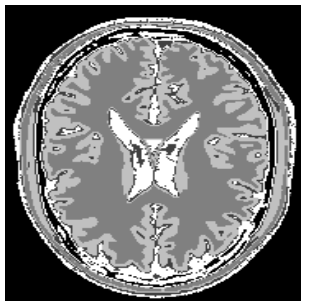

(a)

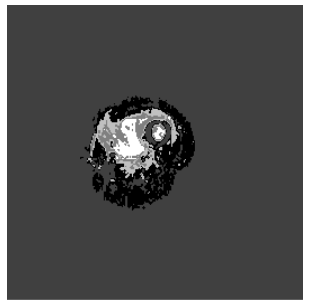

(b)
Fig. 2. Example of HFISA's segmentation results. (a) Segmented T1W1 image with 5 clusters. (b) Segmented STIR image with 5 clusters.

\section{EXPERIMENTS AND RESULTS}

To compare the performance of the proposed algorithm with the standard FCM, we describe the image sets; the parameters of HS and the experiments in the following sections.

\section{A. Data Set}

Since our interest is in medical image analysis, we evaluated our proposed algorithm on two sets of MR images. The first set consists of simulated MR brain images obtained from simulated brain data set of McGill University [13]. The data set contains several subsets of images with varying levels of MRI artifacts; it has a varying noise levels ranging from $0 \%$ to $9 \%$, and varying intensity inhomogeneity varying from $0 \%$ to $40 \%$. Those artifacts make the mission of segmentation is more difficult, therefore reflecting on segmentation results. The second set consists of osteosarcoma MR images provided by the Radiology Department at USM Hospital, Kelantan, Malaysia. These MR images consist of four sequences, T1WI, T2WI, STIR, and T1-post contrast. Fig. 1 shows an examples of these images.

\section{B. HS Parameters Analysis}

In order to obtain the best results from any optimization algorithm, the appropriate selection of the algorithm's parameter values is important, since these parameters will seriously affect the algorithm performance and accuracy. Therefore, the choosing of HS related parameters (i.e. HM, HMCR,PAR and NI) is an important step. Table I show 6 cases tested, each of them with different combinations of HS parameters. These parameter values are selected based on empirical suggestions provided in [21], [28] and the recommended range for HMCR $\in[0.7,0.95]$ and PAR $\in[0.1,0.5]$ as suggested by Yang in
TABLE I

THE HS ALGORITHM PARAMETERS USED IN THE PARAMETERS' ANALYSIS

\begin{tabular}{|c|c|c|c|}
\hline Case & HM & HMCR & PAR \\
\hline 1 & 10 & 0.8 & 0.3 \\
\hline 2 & 10 & 0.9 & 0.45 \\
\hline 3 & 20 & 0.9 & 0.3 \\
\hline 4 & 20 & 0.9 & 0.4 \\
\hline 5 & 30 & 0.8 & 0.3 \\
\hline 6 & 30 & 0.9 & 0.45 \\
\hline
\end{tabular}

TABLE II

OBTAINED RESULTS IN THE PARAMETERS' ANALYSIS FOR EACH CASE

\begin{tabular}{|c|c|c|c|c|c|}
\hline Case & $\begin{array}{c}\text { Obj.Func. } \\
\text { value }\end{array}$ & $\begin{array}{c}\text { no. of } \\
\text { iter. }\end{array}$ & $\begin{array}{c}\text { Cluster } \\
\text { Cent.1 }\end{array}$ & $\begin{array}{c}\text { Cluster } \\
\text { Cent.2 }\end{array}$ & $\begin{array}{c}\text { Cluster } \\
\text { Cent.3 }\end{array}$ \\
\hline 1 & 3328672.75 & 4370 & 28479.67 & 7775.34 & 46481.55 \\
\hline 2 & 3328997.75 & 4988 & 28772.88 & 7773.98 & 46499.56 \\
\hline 3 & 3328869.00 & 3261 & 27213.15 & 7555.07 & 45119.11 \\
\hline 4 & 3328746.50 & 4655 & 28502.98 & 7767.87 & 46565.96 \\
\hline 5 & 3328755.75 & 4592 & 28537.02 & 7767.336 & 46493.71 \\
\hline 6 & 3328838.25 & 4118 & 27245.78 & 7555.252 & 45180 \\
\hline
\end{tabular}

[29]. In this work, the maximum number of iterations (NI) is set to 5000 . The obtained results for each case can be seen in Table II.

As can be seen in Table II, the minimum objective function value is in Case 1 after 4370 iterations, whereas maximum objective function is obtained through Case 2 after 4988 iterations. It should be noted that in some cases better results can be obtained by increasing the maximum number of HS iterations (NI). It can also noted that the values of cluster centers 1,2 and 3 fall within a very narrow range, indicating that the HS algorithm, in effect has found near-optimal solutions despite varying parameter settings. Subsequently, HS parameters are set as per Case 1 values for all experiments.

\section{Experiments and Discussion}

The objective of these experiments is to measure the quality of our proposed approach. In order to do that, HS algorithm is run and then its results (near-optima cluster centers) are fed into the FCM, which we denote FCM/HS. For comparison, we used an FCM with random initialization, which we denote FCM/RAN. We used the objective function value as an indicator of clustering goodness as in [7], [18]. The results for FCM/RAN, and FCM/HS reported here are the average results from 50 trials. The code was written using Matlab version 2008a, and the experiments were performed on Intel core 2 due $2.66 \mathrm{GHz}$ processor with $2 \mathrm{~GB}$ of RAM.

Table III shows 10 cases tested, each of which is an image with different features such as osteosarcoma images (T1, T1 post contrast, T2, STIR) and simulated T1 brain images with different levels of noise and intensity inhomogeneity.

Most of the tested images exhibit significant improvements in terms of minimization of objective function values when using our proposed algorithm FCM/HS as compared to FCM/RAN. However, there are still some images do not show any improvement. Those results motivate us to use another goodness measurement and test the efficiency of using 
TABLE III

RESULTS OF HS, FCM/HS, FCM/RAN (BOLD ENTRIES INDICATE EQUAL OR BETTER FCM/HS THAN FCM/RAN)

\begin{tabular}{|c|c|c|c|}
\hline Image name & $\begin{array}{l}\text { Min Rm } \\
\text { by HS } \\
( \pm \mathrm{SD})\end{array}$ & $\begin{array}{c}\text { Average Rm } \\
\text { FCM/HS } \\
( \pm \mathrm{SD})\end{array}$ & $\begin{array}{c}\text { Average Rm } \\
\text { FCM/RAN } \\
( \pm \mathrm{SD})\end{array}$ \\
\hline $\begin{array}{c}\text { osteosarcoma } \\
\text { STIR }\end{array}$ & $\begin{array}{c}25981.0879 \\
(1.3567)\end{array}$ & $\begin{array}{c}69124431.9006 \\
(0.046105)\end{array}$ & $\begin{array}{c}69124432.0625 \\
(0.27538)\end{array}$ \\
\hline $\begin{array}{c}\text { osteosarcoma } \\
\mathrm{T} 1\end{array}$ & $\begin{array}{l}2360.2505 \\
(0.24939)\end{array}$ & $\begin{array}{c}3103493.3824 \\
(0.019487)\end{array}$ & $\begin{array}{c}3419669.9351 \\
(375323.67)\end{array}$ \\
\hline $\begin{array}{l}\text { osteosarcoma } \\
\text { T1_POST }\end{array}$ & $\begin{array}{c}1644.8068 \\
(0.1739)\end{array}$ & $\begin{array}{c}\text { 7729534.0759 } \\
(0.13883)\end{array}$ & $\begin{array}{c}7729535.4769 \\
(1.0171)\end{array}$ \\
\hline $\begin{array}{c}\text { osteosarcoma } \\
\text { T2 }\end{array}$ & $\begin{array}{l}1255.5207 \\
(0.32201)\end{array}$ & $\begin{array}{c}14013096.886 \\
(1.4817)\end{array}$ & $\begin{array}{c}14013126.423 \\
(36.2472)\end{array}$ \\
\hline $\begin{array}{l}\text { brain T1:ns_0 } \\
\text { inst_inho.0 }\end{array}$ & $\begin{array}{c}1352.3904 \\
(0.51478)\end{array}$ & $\begin{array}{c}\text { 1580519.1135 } \\
(0.16504)\end{array}$ & $\begin{array}{c}1628955.4604 \\
(89217.228)\end{array}$ \\
\hline $\begin{array}{c}\text { brain T1:ns_0 } \\
\text { inst_inho.40 }\end{array}$ & $\begin{array}{l}1356.1311 \\
(0.42575)\end{array}$ & $\begin{array}{c}1942903.095 \\
(0.0864927)\end{array}$ & $\begin{array}{c}1852459.7051 \\
(84323.014)\end{array}$ \\
\hline $\begin{array}{l}\text { brain T1:ns_3 } \\
\text { inst_inho.0 }\end{array}$ & $\begin{array}{l}1340.4924 \\
(0.53404)\end{array}$ & $\begin{array}{c}1671270.6998 \\
(0.033914)\end{array}$ & $\begin{array}{c}1696651.4828 \\
(32748.789)\end{array}$ \\
\hline $\begin{array}{c}\text { brain T1:ns_3 } \\
\text { inst_inho. } 20\end{array}$ & $\begin{array}{l}1312.2024 \\
(0.62517)\end{array}$ & $\begin{array}{c}1770543.9954 \\
(0.046984)\end{array}$ & $\begin{array}{c}1749295.5593 \\
(25223.483)\end{array}$ \\
\hline $\begin{array}{l}\text { brain T1:ns_5 } \\
\text { inst_inho.0 }\end{array}$ & $\begin{array}{l}1293.5919 \\
(0.53934)\end{array}$ & $\begin{array}{c}1835564.2976 \\
(0.23501)\end{array}$ & $\begin{array}{c}1827490.2849 \\
(8836.799)\end{array}$ \\
\hline $\begin{array}{l}\text { brain T1:ns_5 } \\
\text { inst_inho.40 }\end{array}$ & $\begin{array}{l}1279.1602 \\
(0.51645)\end{array}$ & $\begin{array}{c}1835466.0954 \\
(0.52215)\end{array}$ & $\begin{array}{c}1835466.6183 \\
(0.84529)\end{array}$ \\
\hline
\end{tabular}

objective function as a measurement of goodness for clustering results.

Here, we use cluster validity index as a performance metric. Cluster validity index normally used in measuring the quality of clustering algorithms [30]. In this study, three of the most popular cluster validity indexes in fuzzy clustering algorithms were employed, which are partition coefficient (PC) [2], partition entropy coefficient (PE) [31] and Xie_Beni index (XB) [32]. Minimizing the values of $\mathrm{XB}$ and $\mathrm{PE}$ indexes is required while maximizing $\mathrm{PC}$ index values in required. This minimization or maximization of these indexes reveals the quality of clustering. Figures in a Table IV illustrate the results in terms of using these cluster validity indexes as quality measurements. Each case is results of (FCM/RAN) and $(\mathrm{FCM} / \mathrm{HS})$ respectively.

To analyze the results in Table IV, we can categorize the results into 3 groups. The first group of results has almost equal objective function values between (FCM/HS) and (FCM/RAN) as in cases $1,3,4 \& 10$. The second group of results has smaller objective function values for (FCM/HS) as compared to (FCM/RAN) as in cases $2,5 \& 7$. Whereas, the last group of results indicate that objective function values of (FCM/HS) are greater than (FCM/RAN) which is worst in quality measurements as in cases $6,8 \& 9$. Moreover, the last group of results is similar to the scenario in our first experiment displayed in Table III, which is the case of no improvements in objective function values using (FCM/HS) compared to the results obtained by (FCM/RAN).

The first group is a normal case where equal values of objective function obtained by (FCM/HS) and (FCM/RAN) should indicate equal values for results obtained by cluster validity indexes, which is the case that we got. In the second group, when the values of objective function obtained by
(FCM/HS) is less than the values obtained by (FCM/RAN) which is better in terms of objective function that we used, the results obtained by cluster validity indexes indicate in the same direction where the values of cluster validity in (FCM/HS) is better than (FCM/RAN) which is also the case that we got. In the last group the situation is different, according to the results obtained from (FCM/HS) that are in this group greater than results obtained by (FCM/RAN) in term of objective function which are worst in this case. The validity indexes results show opposite results, these results show that results from $(\mathrm{FCM} / \mathrm{HS})$ is not worst, in contrary, it is better than results from (FCM/RAN) in terms of validity index.

To illustrate the idea, in case 6 , the objective function value for (FCM/HS) is 1942900.98 and the objective function value for (FCM/RAN) is 1775411.62 ; this means according to the objective function values that the clustering output of (FCM/RAN) is better that (FCM/HS) while this is not the situation according to cluster validity indexes that show opposite results.

As a result, the objective function values are not necessarily a good indicator of clustering quality in all the times. Therefore, the results obtained from our proposed algorithm are better than the FCM algorithm with random initialization in all types of quality measurements.

\section{HS Execution Time}

The execution time for HS algorithm to find the near optimal cluster centers on MR DICOM image (16 bit) using typical image representation takes about 10 minutes, while the image with a simplified representation took only 10 seconds. This is a significant improvement in execution time. This simplified representation makes our proposed clustering algorithm more efficient.

\section{CONCLUSION}

We proposed a FCM-based image segmentation approach that uses harmony search algorithm to find near-optimal cluster centers. These cluster centers are used as initial centroids for a fuzzy c-means clustering algorithm. The proposed algorithm overcomes FCM's most serious limitations; i.e. sensitivity of cluster centers and its tendency to get trapped in local optima. We then validate our results on an image segmentation problem. Results from the simulated MR brain images and Osteosarcoma images show the superiority of our algorithm over the randomly initialized FCM algorithm. Furthermore, the simplified representing of MR images increases the speed of harmony search to only 10 second per each image. Table IV shows the using of objective function values as a quality measurement could not be always efficient. Therefore, using of cluster validity indexes is encouraged in this type of studies.

\section{ACKNOWLEDGMENT}

This research is supported by 'Universiti Sains Malaysia Research University Grant' grant titled 'Delineation and visualization of Tumour and Risk Structures - DVTRS' under grant number 1001 / PKOMP / 817001. 
TABLE IV

COMPARATIVE RESULTS FROM DIFFERENT VALIDITY INDEXES AND OBJECTIVE FUNCTION.

\begin{tabular}{|c|c|c|c|c|c|}
\hline $\begin{array}{l}\mathscr{D} \\
\tilde{J} \\
\tilde{U}\end{array}$ & Image name & $\mathrm{PC}$ & PE & $\mathrm{XB}$ & Obj_fun \\
\hline \multirow{2}{*}{1} & $\begin{array}{l}\text { osteosarcoma } \\
\text { stir_RAN }\end{array}$ & 0.9514 & 0.1000 & 0.0156 & 69124427.24 \\
\hline & $\begin{array}{l}\text { osteosarcoma } \\
\text { stir_HS }\end{array}$ & 0.9514 & 0.1000 & 0.0156 & 69124426.93 \\
\hline \multirow{2}{*}{2} & $\begin{array}{l}\text { osteosarcoma } \\
\text { T1_RAN }\end{array}$ & 0.8336 & 0.2885 & 0.9946 & 3856294.60 \\
\hline & $\begin{array}{c}\text { osteosarcoma } \\
\text { T1_HS }\end{array}$ & 0.9686 & 0.0727 & 0.0086 & 3103493.26 \\
\hline \multirow{2}{*}{3} & $\begin{array}{l}\text { osteosarcoma } \\
\text { T1_post_RAN }\end{array}$ & 0.9317 & 0.1488 & 0.0318 & 7729534.08 \\
\hline & $\begin{array}{l}\text { osteosarcoma } \\
\text { T1_post_HS }\end{array}$ & 0.9317 & 0.1488 & 0.0318 & 7729534.08 \\
\hline \multirow{2}{*}{4} & $\begin{array}{l}\text { osteosarcoma } \\
\text { T2_RAN }\end{array}$ & 0.8590 & 0.2561 & 0.1238 & 14013106.80 \\
\hline & $\begin{array}{c}\text { osteosarcoma } \\
\text { T2_HS }\end{array}$ & 0.8609 & 0.2561 & 0.1234 & 14013096.89 \\
\hline \multirow{2}{*}{5} & $\begin{array}{l}\text { brn T1 :nos_0 } \\
\text { InsInh.0_RAN }\end{array}$ & 0.8360 & 0.3365 & 0.1260 & 1679706.08 \\
\hline & $\begin{array}{l}\text { brn T1 :nos_0 } \\
\text { InsInh.0_HS }\end{array}$ & 0.8525 & 0.3059 & 0.0525 & 1580516.72 \\
\hline \multirow{2}{*}{6} & $\begin{array}{l}\text { brn T1 :nos_0 } \\
\text { InsInh.40_RAN }\end{array}$ & 0.8129 & 0.3827 & 0.1082 & 1775411.62 \\
\hline & $\begin{array}{l}\text { brn T1 :nos_0 } \\
\text { InsInh.40_HS }\end{array}$ & 0.8233 & 0.3629 & 0.0781 & 1942900.98 \\
\hline \multirow{2}{*}{7} & $\begin{array}{l}\text { brn T1 :nos_3 } \\
\text { InsInh.0_RAN }\end{array}$ & 0.8090 & 0.3917 & 0.1474 & 1738060.41 \\
\hline & $\begin{array}{l}\text { brn T1 :nos_3 } \\
\text { InsInh.0_HS }\end{array}$ & 0.8342 & 0.3459 & 0.0700 & 1671270.24 \\
\hline \multirow{2}{*}{8} & $\begin{array}{l}\text { brn T1 :nos_3 } \\
\text { InsInh.20_RAN }\end{array}$ & 0.8062 & 0.3974 & 0.1281 & 1719949.30 \\
\hline & $\begin{array}{l}\text { brn T1 :nos_3 } \\
\text { InsInh.20_HS }\end{array}$ & 0.8250 & 0.3637 & 0.0762 & 1770542.85 \\
\hline \multirow{2}{*}{9} & $\begin{array}{l}\text { brn T1 :nos_5 } \\
\text { InsInh.0_RAN }\end{array}$ & 0.7860 & 0.4370 & 0.1554 & 1818009.59 \\
\hline & $\begin{array}{l}\text { brn T1 :nos_5 } \\
\text { InsInh.0_HS }\end{array}$ & 0.8070 & 0.3992 & 0.0869 & 1835563.51 \\
\hline \multirow{2}{*}{10} & $\begin{array}{l}\text { brn T1 :nos_5 } \\
\text { InsInh.40_RAN }\end{array}$ & 0.7867 & 0.4369 & 0.0985 & 1835464.00 \\
\hline & $\begin{array}{l}\text { brn T1 :nos_5 } \\
\text { InsInh.40_HS }\end{array}$ & 0.7867 & 0.4369 & 0.0984 & 1835463.91 \\
\hline
\end{tabular}

\section{REFERENCES}

[1] A. K. Jain, M. N. Murty, and P. J. Flynn, "Data clustering: a review," ACM Comput. Surv., vol. 31, no. 3, pp. 264-323, 1999.

[2] J. C. Bezdek, Pattern Recognition with Fuzzy Objective Function Algorithms. Kluwer Academic Publishers, 1981.

[3] J. Kang, L. Min, Q. Luan, X. Li, and J. Liu, "Novel modified fuzzy cmeans algorithm with applications," Digital Signal Processing, vol. 19, no. 2, pp. 309-319, 2009.

[4] J. Wang, J. Kong, Y. Lu, M. Qi, and B. Zhang, "A modified fcm algorithm for mri brain image segmentation using both local and nonlocal spatial constraints," Computerized Medical Imaging and Graphics, vol. 32, no. 8, pp. 685-698, 2008.

[5] J. Bezdek, R. Hathaway, M. Sobin, and W. Tucker, "Convergence theory for fuzzy c-means: counterexamples and repairs," IEEE Trans. Syst. Man Cybern., vol. 17, no. 5, pp. 873-877, 1987.

[6] R. J. Hathaway and J. C. Bezdek, "Local convergence of the fuzzy cmeans algorithms," Pattern Recognition, vol. 19, no. 6, pp. 477-480, 1986.

[7] P. M. Kanade and L. O. Hall, "Fuzzy ants and clustering," IEEE Transactions on Systems, Man and Cybernetics,Part A,, vol. 37, no. 5, pp. 758-769, 2007.

[8] E. R. Hruschka, R. J. G. B. Campello, A. A. Freitas, and A. C. P. L. F. d. Carvalho, "A survey of evolutionary algorithms for clustering," IEEE Transactions on Systems, Man and Cybernetics, Part C: Applications and Reviews, in press.
[9] Z. W. Geem, J. H. Kim, and G. Loganathan, "A new heuristic optimization algorithm: harmony search.” SIMULATION, vol. 76, no. 2, pp. 60-68, 2001.

[10] G. Ingram and T. Zhang, Music-Inspired Harmony Search Algorithm. Springer Berlin / Heidelberg, 2009, ch. Overview of Applications and Developments in the Harmony Search Algorithm, pp. 15-37.

[11] K. S. Lee and Z. W. Geem, "A new meta-heuristic algorithm for continuous engineering optimization: harmony search theory and practice," Computer Methods in Applied Mechanics and Engineering, vol. 194, no. 36-38, pp. 3902-3933, 2005.

[12] R. J. Hathaway and J. C. Bezdek, "Optimization of clustering criteria by reformulation," IEEE Transactions on Fuzzy Systems,, vol. 3, no. 2, pp. 241-245, 1995.

[13] Brain web: Simulated brain database. mcconnell brain imaging centre. montreal neurological institute, mcgill university, http://www.bic.mni.mcgill.ca/brainweb.

[14] S. Z. Selim and K. Alsultan, "A simulated annealing algorithm for the clustering problem," Pattern Recognition, vol. 24, no. 10, pp. 1003-1008, 1991.

[15] K. S. Al-Sultan and S. Z. Selim, "A global algorithm for the fuzzy clustering problem," Pattern Recognition, vol. 26, no. 9, pp. 1357-1361, 1993.

[16] K. S. Al-Sultan, "A tabu search approach to the clustering problem," Pattern Recognition, vol. 28, no. 9, pp. 1443-1451, 1995.

[17] K. S. Al-Sultan and C. A. Fedjki, "A tabu search-based algorithm for the fuzzy clustering problem," Pattern Recognition, vol. 30, no. 12, pp. 2023-2030, 1997.

[18] L. O. Hall, I. B. Ozyurt, and J. C. Bezdek, "Clustering with a genetically optimized approach," IEEE Transactions on Evolutionary Computation,, vol. 3, no. 2, pp. 103-112, 1999.

[19] D. Pham, S. Otri, A. Afify, M. Mahmuddin, and H. Al-Jabbouli, "Data clustering using the bees algorithm," pp. -, 2007.

[20] L. Lili, L. Xiyu, and X. Mingming, "A novel fuzzy clustering based on particle swarm optimization," in First IEEE International Symposium on Information Technologies and Applications in Education, ISITAE, 2007, pp. 88-90.

[21] M. T. Ayvaz, "Simultaneous determination of aquifer parameters and zone structures with fuzzy c-means clustering and meta-heuristic harmony search algorithm," Advances in Water Resources, vol. 30, no. 11, pp. 2326-2338, 2007.

[22] R. Forsati, M. Mahdavi, M. Kangavari, and B. Safarkhani, "Web page clustering using harmony search optimization," in Canadian Conference on Electrical and Computer Engineering, CCECE, 2008, pp. 1601-1604.

[23] M. Mahdavi, M. H. Chehreghani, H. Abolhassani, and R. Forsati, "Novel meta-heuristic algorithms for clustering web documents," Applied Mathematics and Computation, vol. 201, no. 1-2, pp. 441-451, 2008.

[24] X. Wang, X. Z. Gao, and S. J. Ovaska, "A hybrid optimization method for fuzzy classification systems," in Eighth International Conference on Hybrid Intelligent Systems, HIS '08,, 2008, pp. 264-271.

[25] M. Malaki, A. P. J., and H. Abolhassani, "A combinatory approach to fuzzy clustering with harmony search and its applications to space shuttle data," 2008.

[26] Z. W. Geem, Music-inspired Harmony Search Algorithm Theory and Applications. Springer Verlag, 2009.

[27] J. C. Bezdek, J. Keller, R. Krisnapuram, and N. R. Pal, Fuzzy Models and Algorithms for Pattern Recognition and Image Processing. Kluwer Academic Publishers, 1999.

[28] K. Lee, Z. Geem, S. Lee, and K. Bae, "The harmony search heuristic algorithm for discrete structural optimization," Engineering Optimization, vol. 37, no. 7, pp. 663-684, 2005

[29] X.-S. Yang, Music-Inspired Harmony Search Algorithm. Springer Verlag, 2009, ch. Harmony Search as a Metaheuristic Algorithm, pp. $1-14$

[30] M. El-Melegy, E. A. Zanaty, W. M. Abd-Elhafiez, and A. Farag, "On cluster validity indexes in fuzzy and hard clustering algorithms for image segmentation," in IEEE International Conference on Image Processing, ICIP, vol. 6, 2007, pp. 5-8.

[31] J. C. Bezdek and R. Ehrlich, "Fcm: The fuzzy c-means clustering algorithm," Comp. Geosci., vol. 10, no. 2, pp. 191-203, 1984.

[32] X. L. Xie and G. Beni, "A validity measure for fuzzy clustering," IEEE Transactions on Pattern Analysis and Machine Intelligence,, vol. 13, no. 8, pp. 841-847, 1991. 